\title{
Multi-Wavelength Switchable Fibre Ring Laser Based on Polarisation Selective Tilted Fibre Gratings Capable of Strain and Temperature Sensing
}

\author{
Pouneh Saffari ${ }^{1}$, Chengbo Mou ${ }^{1}$, Hongyan $\mathrm{Fu}^{2}$, Lin Zhang ${ }^{1}$ and Ian Bennion ${ }^{1}$ \\ ${ }^{1}$ Photonic Research Group, Aston University, Birmingham, B4 7ET, United Kingdom \\ ${ }^{2}$ Zhejiang University, China \\ Email: saffarip@aston.ac.uk
}

\begin{abstract}
Using three fibre gratings with excessively tilted structures in the cavity, we have experimentally demonstrated a multiwavelength switchable erbium-doped fibre ring laser system. The three tilted gratings act as in-fibre polariser and polarisation dependent loss filters to induce the polarisation hole burning effect in the cavity for the operation of the laser at single, double, triple and quadruple wavelengths. The laser system has demonstrated good stability under room temperature conditions and also achieved a high degree of polarization $(\sim 30 \mathrm{~dB})$, high optical signal to noise ratio (up to $63 \mathrm{~dB})$ and high side mode suppression $(\sim 50 \mathrm{~dB})$. The system has also been investigated for temperature and strain sensing by subjecting the seeding fibre Bragg gratings (FBG) to temperature and strain variations. Since the loss band of the polarisation dependent loss filter is broader than the bandwidth of the seeding FBG, the laser output shifts in wavelength with the applied temperature and strain. The fibre ring laser has shown good responses to the temperature and strain, providing sensitivities of approximately $11.7 \mathrm{pm} /{ }^{\circ} \mathrm{C}$ and $0.85 \mathrm{pm} / \mu \varepsilon$ respectively.
\end{abstract}

Keywords: tilted fibre grating, in-fibre polariser, polarisation dependent loss, fibre ring laser, multi-wavelength switchable operation, temperature and strain sensing.

\section{INTRODUCTION}

Multi-wavelength fibre lasers have potential in a range of different applications, such as wavelength division multiplexed (WDM) fibre communications, optical fibre sensing and instrumentation, and system diagnostics ${ }^{1-4}$. However, due to the low birefringence of standard telecom and Er-doped active fibres, the output of a fibre laser in general is not in a single polarisation state. Numerous techniques have been developed to achieve a single polarisation operation for fibre lasers. Examples can be referred to integrating an integral polariser ${ }^{5}$ and incorporation of a high birefringence rare-earth doped fibre with two anisotropic fibre Bragg gratings (FBGs) ${ }^{6}$. Although these techniques can provide a very high degree of single polarisation oscillation, they suffer from difficulties in fabrication and may induce a high insertion loss to the laser cavity leading to low efficiency. The advantages of intrinsic fibre compatibility and low cost fabrication of FBGs in single mode fibres make them the ideal wavelength selective devices for fibre lasers. Many studies have focused on the operation mechanism of multi-wavelength fibre lasers such as an Er-doped fibre (EDF) ring laser with two cavities ${ }^{7}$, a coupled dual cavity fibre laser ${ }^{8}$, and a fibre laser with a twin-peak reflection grating ${ }^{9}$. In such laser systems, the EDF is the primary gain medium, however, because of the homogenous broadening nature of the EDF, it is difficult to obtain simultaneous or switchable wavelength oscillation in an EDF laser. Furthermore, the homogenous gain broadening of the EDF will result in wavelength competition, even cease the laser oscillation.

In the work we report here, we have employed three fibre gratings inscribed in single mode fibre with excessively tilted structures - one is tilted at $45^{\circ}$ (named as $45^{\circ}$-TFG) and the other two at $77^{\circ}\left(77^{\circ}-\mathrm{TFG}\right)$ and at $81^{\circ}\left(81^{\circ}-\mathrm{TFG}\right)$ - as one in-fibre polariser and two polarisation loss dependent filters, respectively, to realise the single polarisation and multiwavelength switchable operation for an EDF fibre ring cavity laser system. We have achieved single, double, triple and quadruple wavelength oscillation at room temperature condition. An optical signal to noise ratio higher than $50 \mathrm{dBm}$ has been obtained for all lasing wavelengths. In addition, because the bandwidths of the seeding FBGs (normal structure gratings), are narrower than the loss bands of the tilted gratings, the laser output signal has a certain tenability which can

Optical Sensors 2009, edited by Francesco Baldini, Jiri Homola, Robert A. Lieberman, Proc. of SPIE Vol. 7356,

73561S · C 2009 SPIE · CCC code: 0277-786X/09/\$18 · doi: 10.1117/12.820659 
be utilised for optical sensing. We have performed the temperature and strain sensing experiment by subjecting one of the seeding FBGs under the temperature and strain variation. High resolution temperature and strain sensing over a relatively large dynamic range has been demonstrated using this system.

\section{FABRICATION AND CHARACTERISATION OF FIBRE GRATINGS USED IN THE FIBRE RING LASER SYSTEM}

According to previous work ${ }^{10}$, a specially designed $45^{\circ}$-TFG exhibits an ideal in-fibre polariser function as it can provide a strong polarisation dependent loss (PDL) $\sim 30 \mathrm{~dB}$ over nearly $80 \mathrm{~nm}$ bandwidth in the $1550 \mathrm{~nm}$ region. The $45^{\circ}-\mathrm{TFG}$ used in our fibre ring laser system was UV-inscribed in the hydrogen loaded $\mathrm{B} / \mathrm{Ge}$ single mode fibre using scanning phase mask technique. The period of the employed phase mask was $1800 \mathrm{~nm}$. To achieve the tilted fringes at $45^{\circ}$ in the fibre core and induce a radiation response around the $1550 \mathrm{~nm}$ region, the phase mask was rotated at $33.7^{\circ}$ respecting to the fibre axis in the UV-inscription. Limited by the size of the phase mask, concatenation method was used in the fabrication to obtain a $\sim 5 \mathrm{~cm}$ long $45^{\circ}$-TFG. The PDL profile of this grating is very similar to the one shown in ref [10], giving a $28 \mathrm{~dB}$ polarisation extinction ratio from $1480 \mathrm{~nm}$ to $1560 \mathrm{~nm}$.

The other two tilted gratings were fabricated in order to give strong polarisation dependent loss around the four designed seeding wavelengths. According to our design, two gratings with $77^{\circ}$ and $81^{\circ}$ tilted structures may be used as the polarisation dependent loss filters to match the wavelengths of the four seeding FBGs in $1550 \mathrm{~nm}$ region. Thus, the $77^{\circ}$ TFG and $81^{\circ}$-TFG were UV-inscribed in hydrogen loaded standard telecom (SMF-28) fibre using a custom-design phase mask with a $6.6 \mu \mathrm{m}$ period. The mask was carefully rotated at $73^{\circ}$ and $79^{\circ}$ in the inscription system to obtain the desired tilted grating structures.

Figures 1(a) and 2(a) depict the transmission spectra of the $77^{\circ}$ - and $81^{\circ}$-TFG measured in the wavelength region from $1200 \mathrm{~nm}$ to $1700 \mathrm{~nm}$. From the figures we can clearly see there is pronounced peak splitting on the spectra and all the peaks are relatively weak giving strengths around 2-3dB. These two spectra were measured using unpolarised light. When we inserted a polariser and a polarisation controller between the broadband source and the TFG, either split peak was excited depending on the polarisation of the probe light. As clearly shown in Figure 1(b) and 2(b), which give two zoomed split-peaks for $77^{\circ}$-TFG and $81^{\circ}$-TFG, respectively, we see that when the light switched from the fast-axis polarisation to the slow-axis one, the peaks at the shorter wavelength sides vanish while the peaks at the longer wavelength side grow to maximum and vice versa. This evidently indicates that the $77^{\circ}$-TFG and $81^{\circ}$-TFG are strong polarisation loss dependent filters and this property can be utilised to induce wavelength selective polarisation-holeburning (PHB) effect to realise multi-wavelength switchable function in proposed fibre ring laser system.

In the proposed fibre ring laser system, four normal structure FBGs were used as seeding wavelengths and fed to the ring cavity via an optical circulator as shown in Figure 3. These four FBGs were fabricated in hydrogen loaded SMF-28 fibre using standard UV-inscription and the phase mask technique. The Bragg wavelengths and reflectivities for the four FBGs were $1547 \mathrm{~nm} / 2.5 \mathrm{~dB}, 1553 \mathrm{~nm} / 2.2 \mathrm{~dB}, 1563 \mathrm{~nm} / 2.1 \mathrm{~dB}$ and $1569 \mathrm{~nm} / 5.6 \mathrm{~dB}$, respectively. These four wavelengths were chosen to match the four polarisation loss bands of the $77^{\circ}$-TFG and $81^{\circ}$-TFG in $1550 \mathrm{~nm}$ region. All tilted and normal structure gratings were thermally annealed at $80^{\circ} \mathrm{C}$ for 48 hours after the fabrication to stabilise their spectral property. 

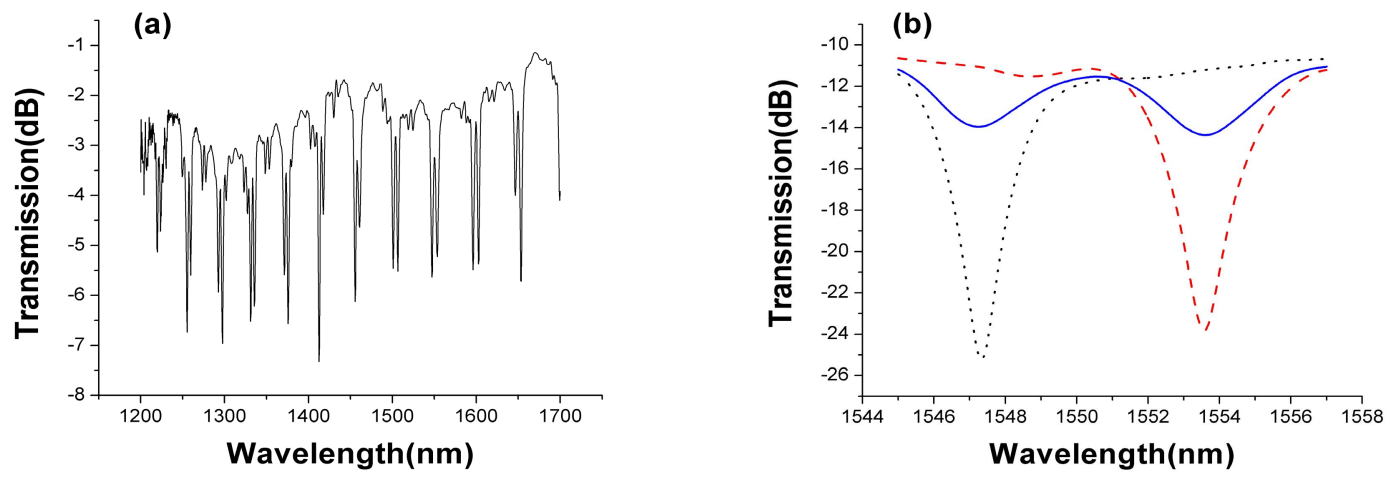

Figure.1. (a) Transmission spectrum of the $77^{\circ}-\mathrm{TFG}$ over wavelength range of $1200 \mathrm{~nm}-1700 \mathrm{~nm}$; (b) Zoomed spectra of one paired polarisation loss peaks of $77^{\circ}-\mathrm{TFG}$ around $1550 \mathrm{~nm}$ measured with randomly (solid line) and fully polarised input lights (dashed lines).
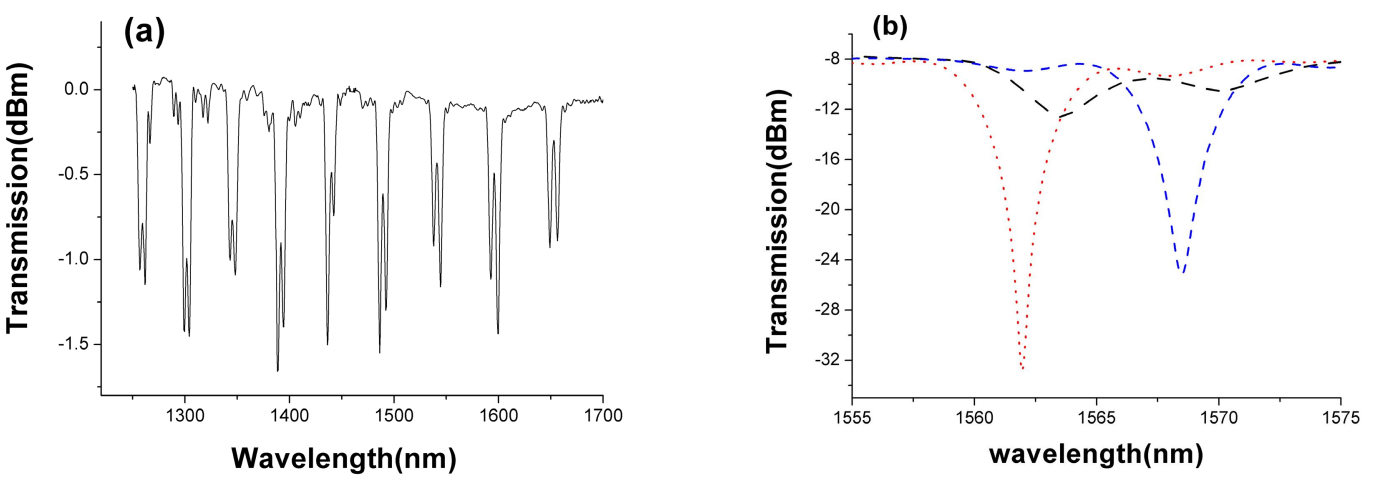

Figure.2. (a) Transmission spectrum of the $81^{\circ}$-TFG over wavelength range of $1200 \mathrm{~nm}-1700 \mathrm{~nm}$; (b) Zoomed spectra of one paired polarisation loss peaks of $81^{\circ}-\mathrm{TFG}$ around $1550 \mathrm{~nm}$ measured with randomly (solid line) and fully polarised input lights (dashed lines).

\section{OPERATION PRINCIPLE OF THE MULTI-WAVELENGTH SWITCHABLE FIBRE RING LASER}

Figure. 3 shows the set-up of the proposed multi-wavelength switchable fibre ring laser system. In this configuration, the gain medium is a $10 \mathrm{~m}$ section of highly erbium doped fibre, which is pumped by a $975 \mathrm{~nm}$ laser diode through a $980 / 1550$ WDM coupler. An optical isolator (OIS) ensures an anticlockwise ring cavity. The $30 \%$ arm of the coupler is used as the output port of the laser. The $77^{\circ}-\mathrm{TFG}$ and $81^{\circ}$-TFG behave as the polarisation dependent spectral loss filters and the $45^{\circ}$ - TFG is used as an in-fibre polariser in the system to ensure the laser operating in a single polarisation state and wavelength switchable regime. 


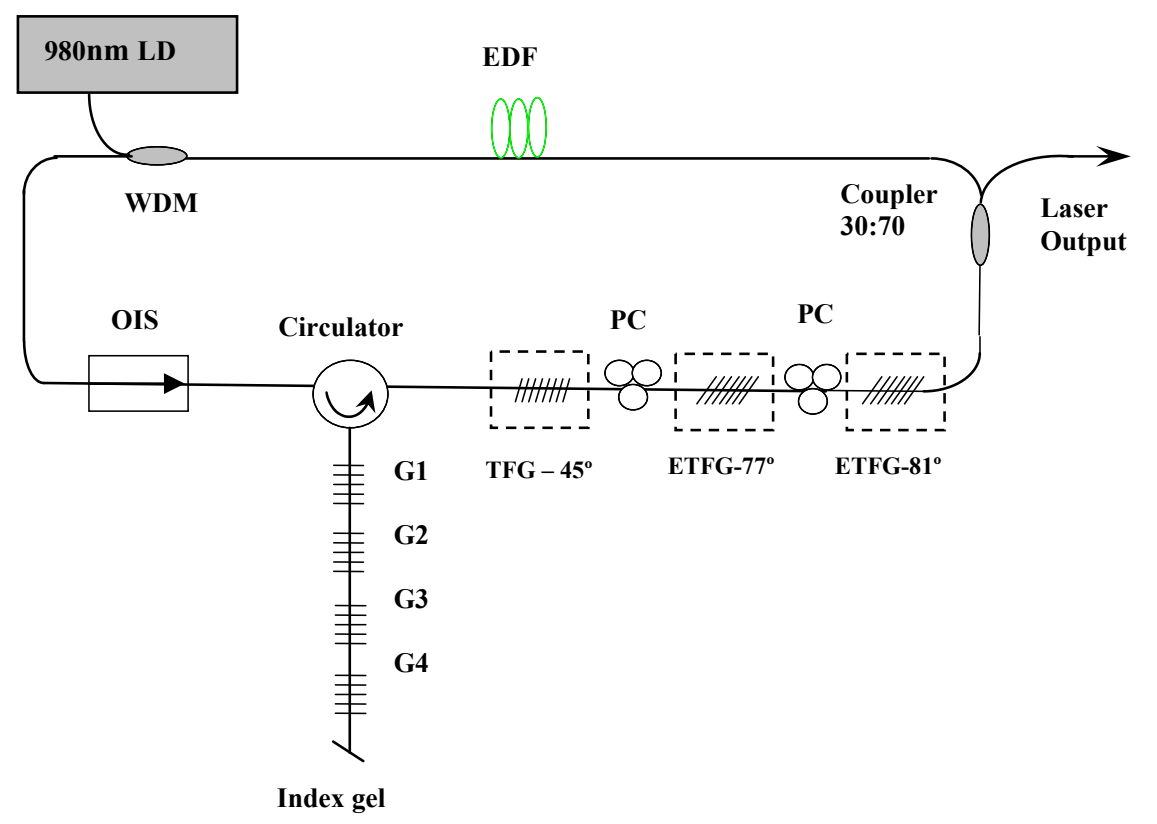

Figure.3. Schematic diagram of the experimental setup for the multi-wavelength switchable fibre ring laser.

Two fibre polarisation controllers (PCs), one placed between $45^{\circ}$-TFG and $77^{\circ}$-TFG and the other placed between $77^{\circ}-\mathrm{TFG}$ and $81^{\circ}-\mathrm{TFG}$, are employed in the system to adjust the polarisation state of the cavity. The four standard FBGs (G1, G2, G3 and G4), whose wavelengths are matched with the loss bands of the TFG polarisation loss filters, function as seeding wavelength selectors and coupled into the laser cavity via a circulator. This circulator also acts as an isolator to maintain the single direction oscillation. Since the reflection bands of the four seeding FBGs are narrower than the loss bands of the TFGs, the four FBGs can be used as the temperature and strain sensors to give the system sensing capability. The end of the FBG array is terminated by index matching gel in order to eliminate background ASE noise. By introducing PDL, cavity loss can be controlled and wavelength of laser line can be varied. Therefore it is possible to operate the laser giving output at single, double, triple and quadruple wavelength operation by simply adjusting the polarisation state of the light entering the $77^{\circ}$-TFG and $81^{\circ}$-TFG using the PCs. As FBGs are sensitive to the temperature and strain changes the laser wavelength can be tuned continuously by heating and stretching or compressing the FBGs within the range defined by the TFG loss band.

\section{CHARACTERISATION OF THE FIBRE RING LASER}

By adjusting the PCs to control the polarisation state of the light entering the $77^{\circ}$-TFG and $81^{\circ}$-TFG (polarised in the equivalent fast- or slow-axis of the TFGs), single-wavelength lasing at either the four seeding wavelengths has been demonstrated. Figures 4 (a) - (d) evidently show the single wavelength oscillation of the fibre ring laser at the four seeding wavelengths at $1547.05 \mathrm{~nm}, 1553.27 \mathrm{~nm}, 1563.05 \mathrm{~nm}$ and $1568.97 \mathrm{~nm}$ respectively. We have also measured the pumping efficiency for the EDF system just for lasing at $\sim 1553 \mathrm{~nm}$. For this experiment, the output of the ring cavity was connected to an optical spectrum analyser (OSA) first and we then increased the driven current to the $975 \mathrm{~nm}$ pump diode. When the current was below the threshold, we only saw the ASE broadened spectrum on the OSA and, as soon as the current reached the threshold, we saw a narrow lasing peak at $\sim 1553 \mathrm{~nm}$ occurring on the OSA. At this point, the output terminal of the ring laser was disconnected from the OSA and attached to a power meter via a pigtail fibre. We then recorded the output power of the laser for each driving current. Figure 5 plots the laser output against pumping current for the lasing wavelength at $\sim 1553 \mathrm{~nm}$. From the figure we can estimate that the apparent pumping efficiency is about $1.95 \mu \mathrm{W} / \mathrm{mA}$. 
We also verified the single polarisation state of the outputs for this fibre ring laser system by connecting the laser output to a polarisation controller followed by a commercial polariser and a power-meter. The measured degree of polarisation (DOP) was in the range of $30 \mathrm{~dB}(99.80 \%)$ to $35 \mathrm{~dB}(99.94 \%)$ for the lasing oscillation at four different wavelengths, indicating a very high degree of single polarisation operation of the laser system.
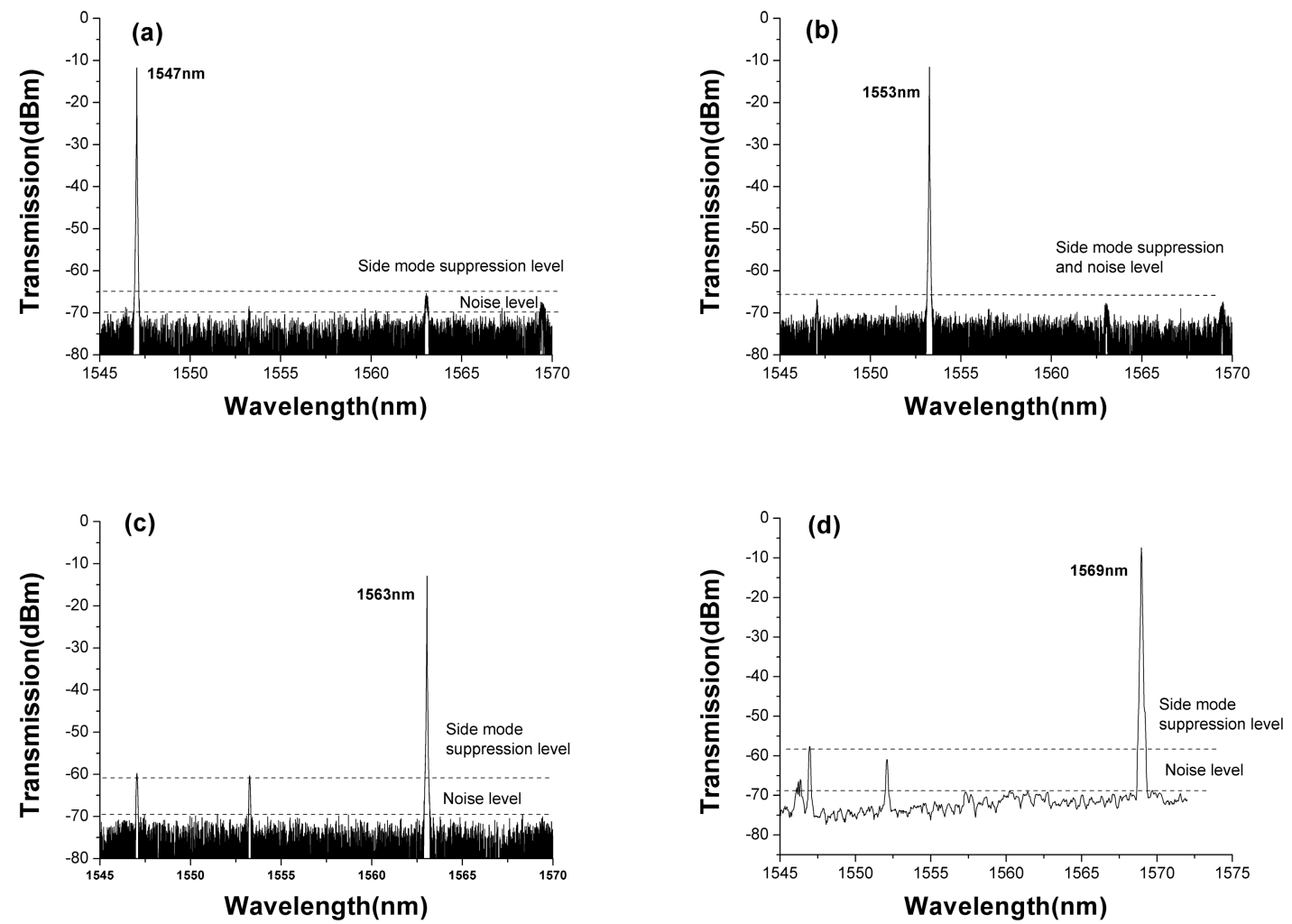

Figure.4. Single wavelength lasing at (a) $1547.05 \mathrm{~nm}$, (b) $1553.27 \mathrm{~nm}$, (c) $1563.05 \mathrm{~nm}$ and (d) $1568.97 \mathrm{~nm}$.

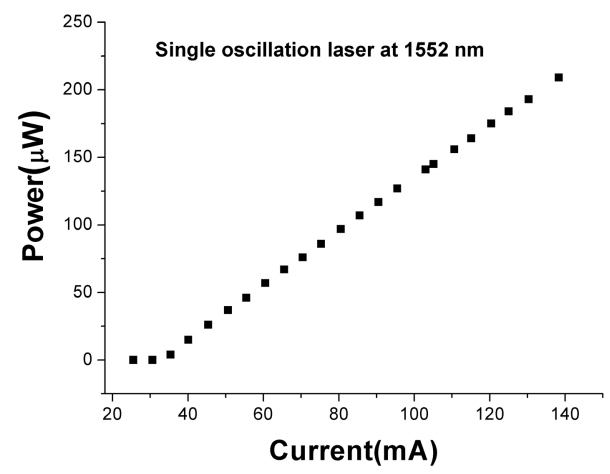

Figure.5. Output power against pump diode current for lasing at $1553 \mathrm{~nm}$.

Similarly, adjusting the two PCs the polarisation state of the light entering the $77^{\circ}$-TFG and $81^{\circ}$-TFG will change. This act results the changes in the PHB profile so the laser oscillation at double, triple and quadruple wavelengths can be 
achieved in this system. Figure 6 (a), (b) and (c) show three sets of dual-wavelength oscillations at $1547.06 \mathrm{~nm} / 1553.27 \mathrm{~nm}, 1547.06 \mathrm{~nm} / 1563.07 \mathrm{~nm}$ and $1547.06 \mathrm{~nm} / 1563.07 \mathrm{~nm}$, respectively. Figure 7 (a) and (b) show the two sets of spectra for triple-wavelength oscillations at $1546.94 \mathrm{~nm} / 1551.99 \mathrm{~nm} / 1562.63 \mathrm{~nm}$ and $1551.99 \mathrm{~nm} / 1562.63 \mathrm{~nm} / 1568.89 \mathrm{~nm}$ and Figure 7 (c) shows the spectrum for one quadruple laser oscillation.
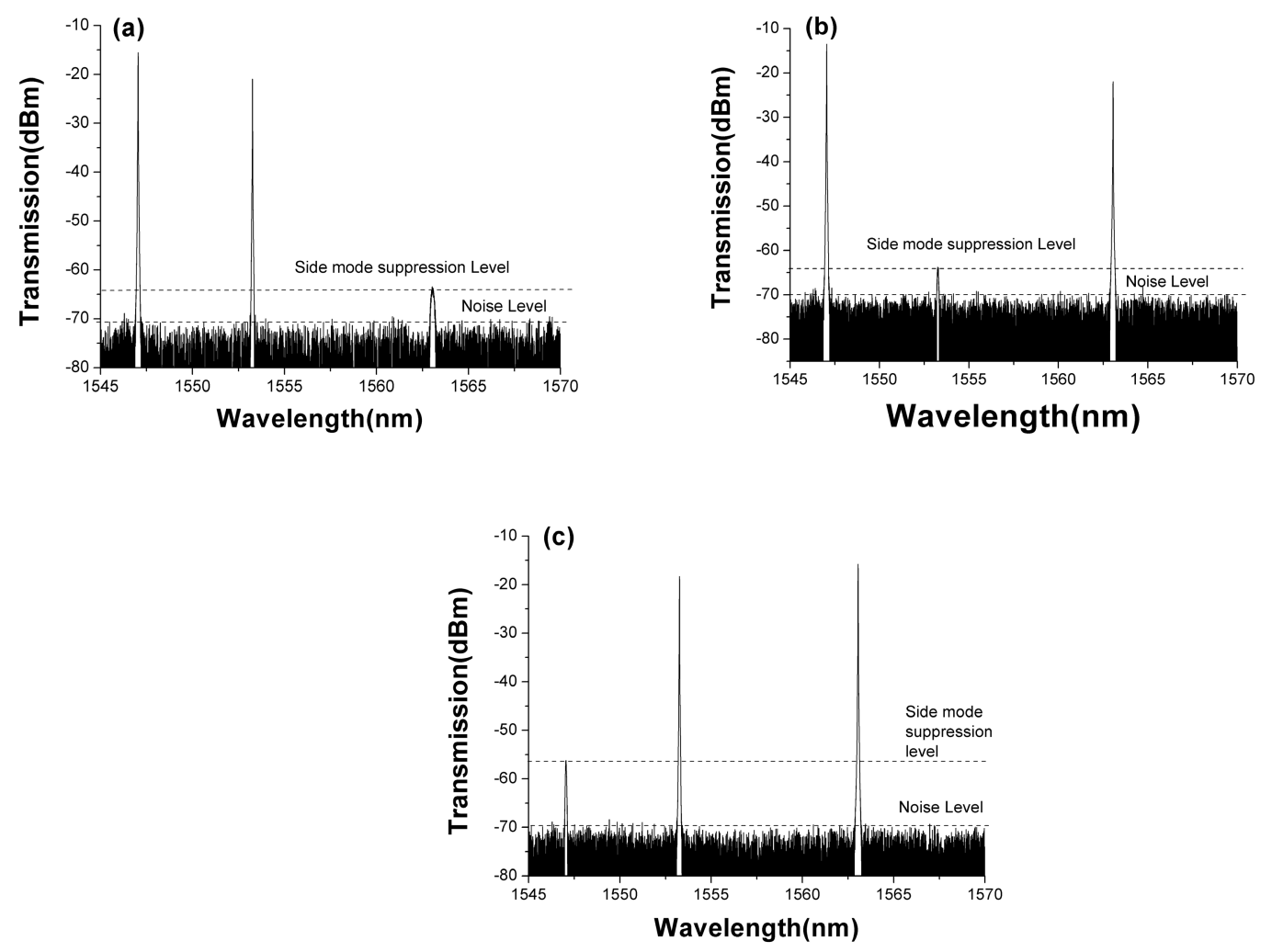

Figure.6. Demonstration of alternative dual-wavelength lasing at (a) $1547 . .06 \mathrm{~nm} / 1553.27 \mathrm{~nm}$ (b) $1547.05 \mathrm{~nm} / 1553.27 \mathrm{~nm}$ and (c) $1553.27 \mathrm{~nm} / 1563.05 \mathrm{~nm}$.
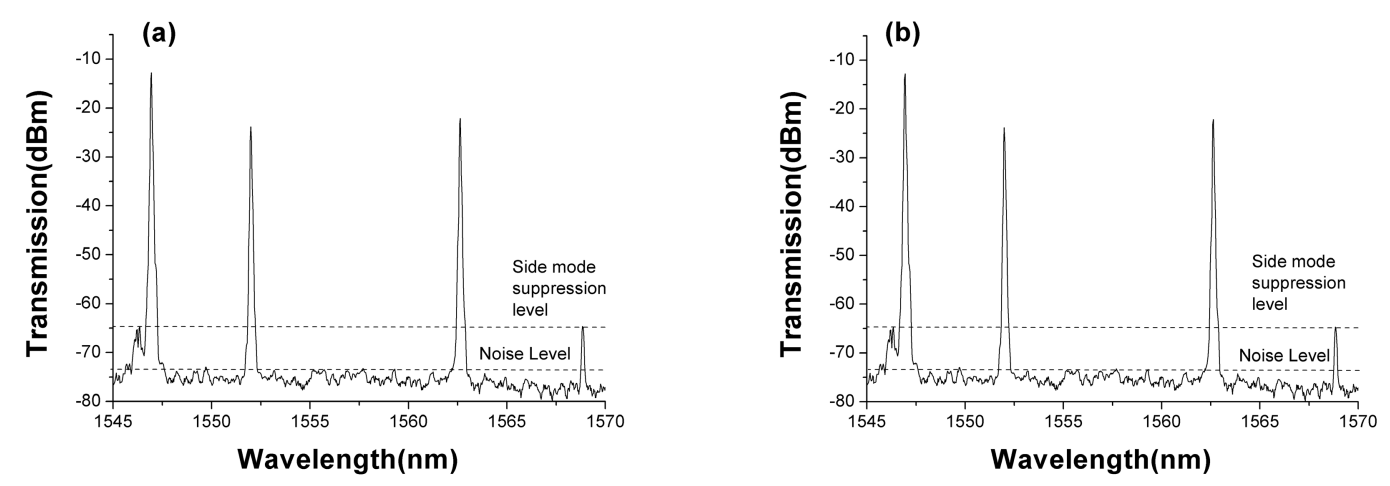

Proc. of SPIE Vol. 7356 73561S-6 


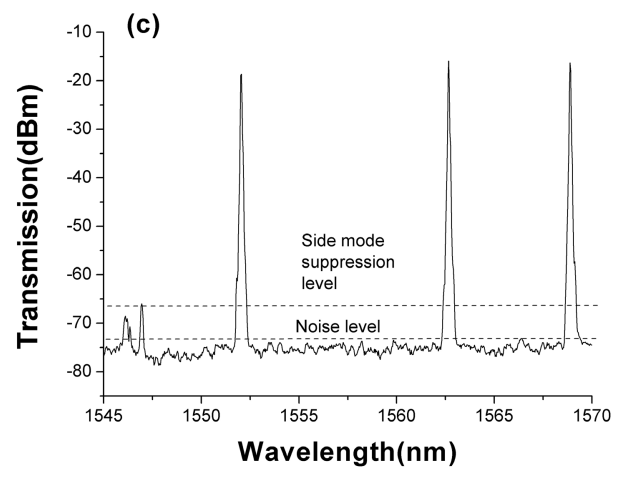

Figure.7. Triple wavelength oscillations at (a) $1546.94 \mathrm{~nm} / 1551.99 \mathrm{~nm} / 1562.63 \mathrm{~nm}$ and (b) $551.99 \mathrm{~nm} / 1562.63 \mathrm{~nm} / 1568.89 \mathrm{~nm}$.

(c) Quadruple wavelength oscillation for all four wavelengths.

All sets of single- and multi-wavelength lasing oscillations have been continuously monitored for 30 minutes in the laboratory at room temperature for stability assessment. No noticeable amplitude fluctuation was observed for the laser operating at single- and multi-wavelengths when the PCs were fixed at their positions during the experiment. Figure.8 is an example of the continuously monitored lasing profiles on the OSA for a dual-wavelength lasing situation, showing no degrading of output amplitude.

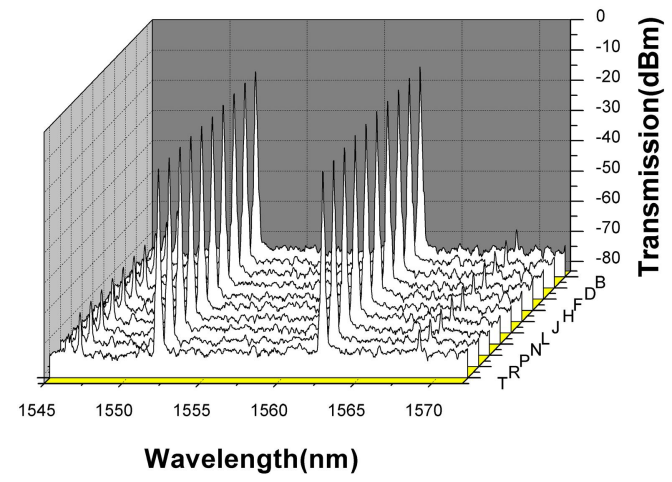

Figure.8. Stability measurements for one of the double-wavelength operations, showing no noticeable variation for the output profile.

From all measurements of single- and mulit-wavelength lasing oscillations, we have estimated the optical signal to noise ratio (OSNR) and side mode suppression. The measurement showed that values of OSNR for single, dual, triple and quadruple lasing lines in our proposed system were in the range between $50 \mathrm{~dB}$ to $63 \mathrm{~dB}$ and the side mode suppression ratio values were in the range from $40 \mathrm{~dB}$ to $55 \mathrm{~dB}$. The summary of these values are presented in Table 1 . 


\begin{tabular}{|c|c|c|c|}
\hline $\begin{array}{c}\text { Oscillation } \\
\text { wavelength }\end{array}$ & $\begin{array}{c}\text { Stability } \\
\text { time }\end{array}$ & $\begin{array}{c}\text { Side mode } \\
\text { suppression } \\
\text { ratio }\end{array}$ & $\begin{array}{c}\text { Optical } \\
\text { signal to } \\
\text { noise } \\
\text { ratio }\end{array}$ \\
\hline Single & $30 \mathrm{~min}$ & $55 \mathrm{~dB}$ & $63 \mathrm{~dB}$ \\
\hline Dual & $30 \mathrm{~min}$ & $50 \mathrm{~dB}$ & $54 \mathrm{~dB}$ \\
\hline Triple & $30 \mathrm{~min}$ & $48 \mathrm{~dB}$ & $53 \mathrm{~dB}$ \\
\hline Quadruple & $30 \mathrm{~min}$ & $40 \mathrm{~dB}$ & $50 \mathrm{~dB}$ \\
\hline
\end{tabular}

Table.1- Summary of the measured technical values of the proposed ring laser cavity.

\section{SENSING CAPABILITY OF THE RING LASER CAVITY}

So far we have only demonstrated single- and multi-wavelength operations at fixed wavelengths. However, as the reflection bands of the seeding FBGs are much narrower than the loss peaks of $77^{\circ}$-TFG and $81^{\circ}$-TFG, the system has the capability of wavelength tuning by tuning the Bragg wavelengths of the seeding gratings within the loss bands of the TFGs. If the seeding FBGs are subjected to temperature and strain variation their wavelengths will shift accordingly.

Considering the above fact, sensing capability of our proposed system was investigated for temperature and strain sensing. For demonstrating the concept, the system was made lasing at single wavelength at $1562.27 \mathrm{~nm}$ and the seeding FBG with central wavelength at $\sim 1562 \mathrm{~nm}$ was then subjected to rising temperatures from $0^{\circ} \mathrm{C}$ to $102^{\circ} \mathrm{C}$ using a temperature controlled heater and we clearly saw that the output of the fibre ring laser system shifted towards the longer wavelength side. Figure 9(a) shows the temperature rising induced laser spectrum evolution and Figure 9(b) plots the laser output wavelength shift against the temperature. From figure $9(\mathrm{~b})$ we estimate a temperature sensitivity of $11.7 \mathrm{pm} /{ }^{\circ} \mathrm{C}$. We then subjected the seeding grating to strain variation using a micrometer fibre stretcher. The strain applied to the seeding grating was from $0 \mu \varepsilon$ to $2324 \mu \varepsilon$. Figure 10 (a) and (b) give the results for strain induced laser spectral evolution and the wavelength shift against the applied strain, respectively. From the slope of the plot in Figure 10 (b), we estimate the strain sensitivity of $0.85 \mathrm{pm} / \mu \varepsilon$ for the device.
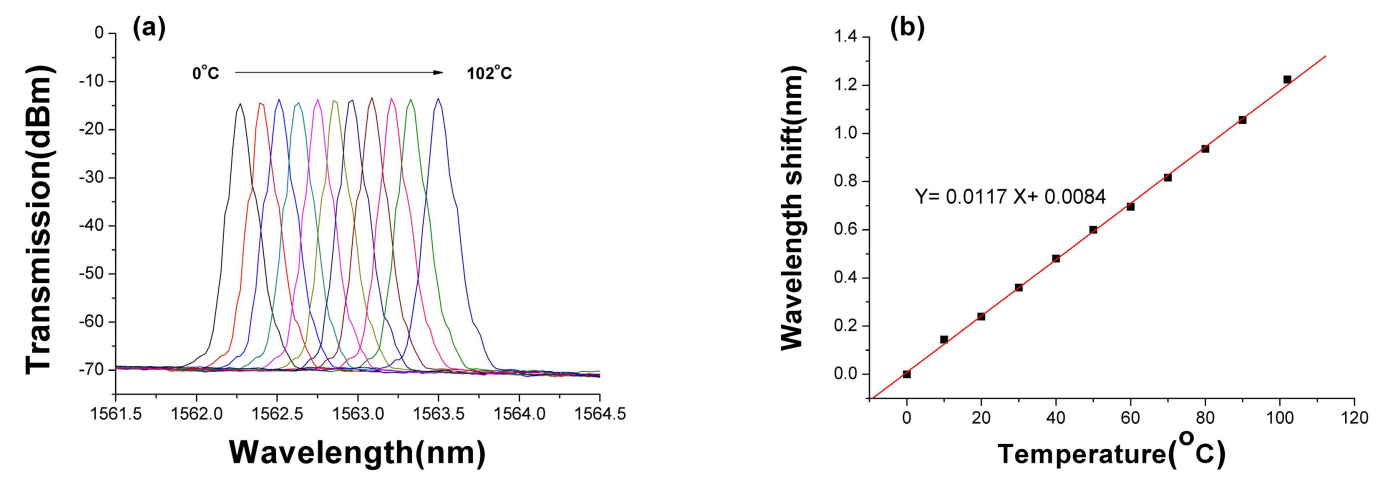

Figure.9. (a) Increasing temperature induced spectral evolution and (b) wavelength shift against temperature. 

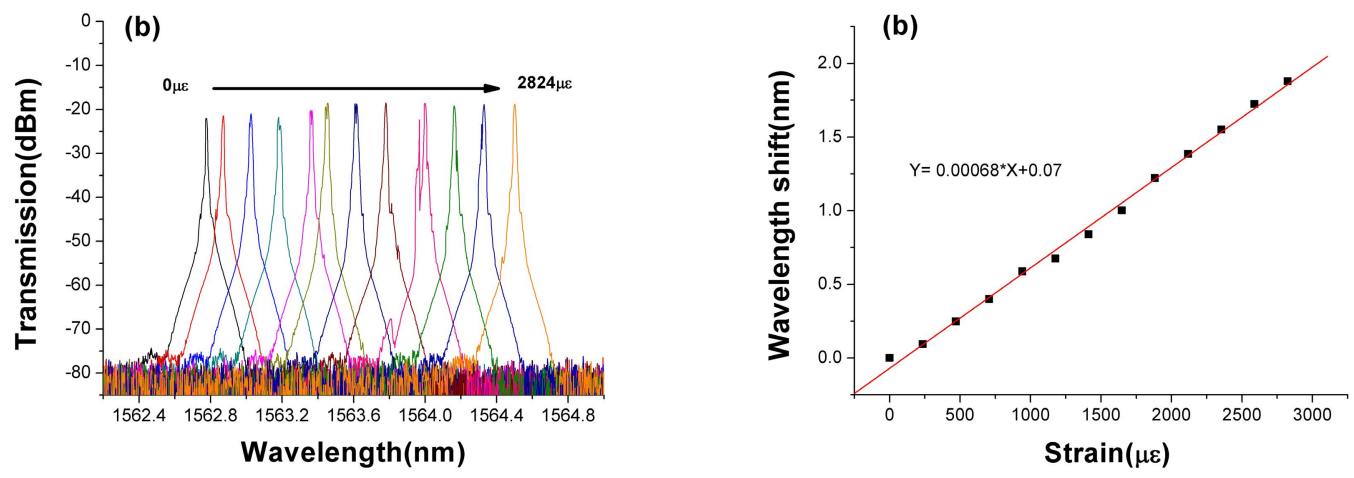

Figure.10. (a) Strain induced spectral evolution and (b) wavelength shift against strain.

\section{SUMMARY}

In conclusion, we have demonstrated a novel, stable, single polarisaton and multi-wavelength switchable fibre ring laser system using three special tilted fibre gratings - $45^{\circ}$-TFG, $77^{\circ}$-TFG and $81^{\circ}$-TFG. The $45^{\circ}$-TFG act as an in-fibre polariser and $77^{\circ}$-TFG and $81^{\circ}$-TFG give polarisation dependent loss in four designated wavelength bands, matching with the four seeding wavelengths. The TFG induced PHB effect allows the fibre ring laser system operating at can single, double, triple and quadruple state by simply adjusting the PCs in the cavity. As all the gratings were made in single mode fibres, the fabrication cost and insertion loss are intrinsically low. The TFG based laser system has also shown good stability and high OSNR (up to 63dB) and efficient side mode suppression (up to 50dB). Finally, we have demonstrated the capability of this system for optical sensing application. Since the loss bandwidth of the TFG is broader than the seeding FBG reflection band, when the seeding FBG is subjected to temperature or strain variation, the laser output wavelength will change. We have heated one of the seeding FBGs from $0^{\circ} \mathrm{C}$ to $102^{\circ} \mathrm{C}$ and also subjected it to the strain change from $0 \mu \varepsilon$ to $2324 \mu \varepsilon$ and investigated its temperature and strain responses. The temperature and strain sensitivity of $11.7 \mathrm{pm} /{ }^{\circ} \mathrm{C}$ and $0.85 \mathrm{pm} / \mu \varepsilon$ respectively were obtained. Since it is laser output, the sensing signal resolution should be much higher if a high resolution demodulation system is used, instead using a standard optical spectrum analyser. The demonstrated multi-wavelength switchable fibre ring laser system may be used as a useful singlepolarisation narrow-line multi-wavelength light source and a high resolution sensing system.

\section{REFERENCES}

[1] Li,H.,Ding,.H and Chan,K.T.,"Erbium- doped fibre lasers for dual wavelength operation," Electron. Lett., vol. 33, pp. 52-53, 1997.

[2] Chow,J.,Town,G.,Eggleton,B.,Ibsen,M.,Sudgen,K. and Bennion,I.,"Multiwavelength generation in erbium-doped fibre laser using in-fibre comb filters," IEEE Photon. Technol. Lett., vol. 8, pp.601-602, 1996.

[3] Das,G.and Lit,J.W.Y.,"L-band multiwavelength fibre laser using an elliptical fibre," IEEE Photon Technol. Lett., vol. 14, pp. 606-608, 2002.

[4] Talaverano,L.,Abad,S.,Jarabo,S.and Lopez-Amo,S.,"Multiwavelength fibre laser sources with Bragg-grating sensor multiplexing capability," J. Lightwave Technol., vol. 19, pp. 553-558, 2001.

[5] Lin,J.,T.and Gambling,W.,A.,"Polarization effects in fibre lasers: phenomena, Theory and Applications," In Fibre Laser Sources and Amplifiers II, vol. 1373 (SPIE 1990), pp. 42-53, 1990.

[6] Pureur,D.,Douay,M.,Bernage,P.,Niay,P.and Bayon,J.F.,"Single-Polarisation Fibre Using Bragg Gratings in Hi-Bi Fibres," IEEE Jour. Light. Techno., vol.13, No. 3, pp. 350-355, 1995. 
[7] Okamura,H.and Iwatsuki,K.,"simultaneous oscillation of wavelength-tunable, singlemode lasers using an Er-doped fibre amplifier," Electron. Lett., vol. 28, pp. 461-463, 1992.

[8] Chernikov,S.V.,Taylor,J.R.and Kashyap,R.,"Coupled-cavity erbium fibre lasers incorporating fibre grating refeltors," Opt. Lett., vol.23, pp. 2023-2025, 1993.

[9] Chemikov,S.V.,Kashyap,R.,Mckee,P.F. and Taylor,J.R.,"Dual frequency all fibre grating laser source," Electron. Lett., vol. 29, pp.1089-1090, 1993.

[10] Zhou,K.,Simpson,G.,Chen,X.,Zhang,L.and Bennion,I.,"High extinction ratio in-fibre polarizer s based $45^{\circ}$ tilted fibre bragg gratings," Opt.Lett. vol.30, pp. 1285-1287, Jun.2005. 